University of Nebraska - Lincoln

DigitalCommons@University of Nebraska - Lincoln

$5-1-2006$

\title{
Multiobjective Plan Selection Optimization for Traffic Responsive Control
}

Montasir M. Abbas

VirginiaTech, abbas@vt.edu

Anuj Sharma

University of Nebraska - Lincoln, anujs@iastate.edu

Follow this and additional works at: https://digitalcommons.unl.edu/civilengfacpub

Part of the Civil Engineering Commons

Abbas, Montasir M. and Sharma, Anuj, "Multiobjective Plan Selection Optimization for Traffic Responsive Control" (2006). Civil Engineering Faculty Publications. 13.

https://digitalcommons.unl.edu/civilengfacpub/13

This Article is brought to you for free and open access by the Civil Engineering at DigitalCommons@University of Nebraska - Lincoln. It has been accepted for inclusion in Civil Engineering Faculty Publications by an authorized administrator of DigitalCommons@University of Nebraska - Lincoln. 


\title{
Multiobjective Plan Selection Optimization for Traffic Responsive Control
}

\author{
Montasir M. Abbas ${ }^{1}$, P.E.; and Anuj Sharma ${ }^{2}$ \\ ${ }^{1}$ Department of Civil and Environmental Engineering, Virginia Tech, Blacksburg, VA, corresponding author: abbas@vt.edu \\ ${ }^{2}$ Department of Civil Engineering, University of Nebraska, Lincoln, NE: sharma3@unl.edu
}

Note: Discussion remained open until October 1, 2006. The manuscript for this paper was submitted for review December 28, 2004; approved May 18, 2005; published May 1, 2006.

Abstract: Optimal coordination of traffic signals requires proper activation of timing plans to match current traffic conditions. One of the greatest challenges in configuring a traffic responsive plan selection control mode is how to select only $n$ number of timing plans (restricted by traffic controller memory limitations) to address possibly all traffic conditions/ states that can be encountered in the field, for a specific site, but most importantly, how to associate each of these traffic conditions to one of the $n$ timing plans stored in the traffic controller. This paper uses a multiobjective nondominated sorting genetic algorithm to (1) select the best $n$ timing plans to be stored in the controller and (2) group traffic states into $\mathrm{m}$ number of clusters, and associate each of the $m$ clusters with one of the stored timing plans. A new performance measure, degree of detachment, developed by the writers to address the clustering requirements, is described in this paper.

CE Database subject headings: Traffic management; Traffic control; Optimization; Algorithms.

\section{Introduction}

Coordinating traffic signals in a closed-loop system can provide significant reductions in travel times and delay. Previous studies have shown that up to $20 \%$ in travel time reduction can be achieved by interconnecting signals and providing newly optimized timing plans (Meyer 1997). Another study evaluating the impact of properly timing a closed loop system in Texas reported a 13.5\% (20.8 million gallons/year) reduction in fuel consumption, a 29.6\% (22 million hours/year) reduction in delay, and an 11.5\% (729 million stops/year) reduction in stops (Fambro et al. 1995). The study estimated total savings to the public of approximately \$252 million in the following year alone. These kinds of benefits, however, require the implementation of timing plans that are most suitable to the existing traffic conditions in the field. This in turn requires that timing plans be varied in a timely manner as the traffic conditions change.

The traffic responsive plan selection (TRPS) control mode provides a mechanism by which the traffic signal system is able to change timing plans in real time in response to changes in traffic conditions. The objective of the TRPS mode is to enable the signal controller to implement timing plans that are optimal for the traffic conditions that currently exist. There are, however, two major challenges in setting up a TRPS system: (1) development/selection of optimal timing plans that are suitable for a wide range of traffic conditions and (2) mapping/association of each traffic condition to one of the few timing plans stored in the traffic controller.

This paper describes how a nondominated sorting genetic algorithm (NSGA-II) was used to select only 14 timing plans (to comply with the controller limitation) out of a total of 10,353 possible plans originally designed with PASSER V software (Chaudhary et al. 2002). The NSGA-II simultaneously changed the selection of the individual timing plans included in the chosen set, as well as the association of each traffic state to one of the timing plans. The 14 timing plans and their state association were optimized such that the overall system delay and number of vehicular stops were minimized.

The methodology presented in this paper was developed as a part of a research that aims at the development of general guidelines for setup of TRPS (Abbas et al. 2004). The paper discusses a comprehensive approach for selection of optimal timing plans. Once the timing plans for a certain network have been chosen, TRPS parameters need to be selected such that the most suitable plan in the controllers' database is selected to match the existing traffic conditions.

\section{Timing Plan Selection for TRPS Control}

There is a very limited amount of research on the optimal selection of timing plans for TRPS control. The city of Milwaukee, Wisconsin, has implemented a TRPS mode in one of their closed-loop systems to manage congestion and reduce traffic accidents (Hanbali and Fornal 1998). The study used only two cycle lengths of 90 and 120 seconds, and it reported an increase in approach capacity and vehicle speed over system detectors. Another study of two networks in Lafayette, Ind., found that TRPS mode reduced total system delay by $14 \%$ compared to total oxygen demand (TOD) mode for midday traffic pattern (Nelson et al. 2000). These studies, however, implemented a very limited number of timing plans that were specifically designed to address specific traffic states. The question that remains unanswered is: how to select a limited number of timing plans to provide an optimal performance with all traffic conditions that can 
be encountered in the field, and not only the limited states in the case study.

\section{State-Timing Plan Space}

The problem of selecting timing plans for traffic responsive operation can be represented by two variables: states $(S)$ and timing plans $(P)$. The state variable describes the existing traffic pattern in all approaches at a certain point in time. The timing plan variable identifies the optimum timing plan for the current state. The main challenges of a TRPS setup are: (1) to select the optimal subset of the $P$ space to be included in the limited memory of the traffic controllers and (2) to determine the optimal plan from the available $P$ space that should be applied to the existing sample of the $S$ space. It can therefore be recognized that for a complete representation and evaluation of the studied system, all state-plan combinations have to be considered and evaluated.

\section{Traffic States Clustering}

In TRPS control, once timing plans are selected and associated with traffic states, the TRPS system parameters are configured to activate a timing plan when its associated state is recognized. The activation mechanism is implemented through a threshold system, where timing plans are activated when certain traffic volumes and/or occupancy exceed or fall below these thresholds. As such, when a timing plan is included in the selected set of timing plans, not only does it need to be associated with different traffic states, but it also needs to be associated with adjacent traffic states. Otherwise, the TRPS threshold mechanism might not be able to activate the timing plan. It is therefore required to cluster the traffic states into groups with common characteristics. In other words, the states clustered together need to be homogeneous in their performance measures with the selected timing plan.

Some of the previous research used $K$-means clustering of traffic states (Sharma 2004). The K-means procedure considers each observation as an object in an $m$-dimensional space. The procedure groups traffic states that are closer in their attributes (east bound volume, north bound volume, etc.) together. However, the K-means clustering procedure does not take into account the delay or number of stops associated with assigning a timing plan to different states. Therefore, applying a K-means clustering will merely result in forming $\mathrm{m}$ number of homogeneous states in terms of traffic volume itself, but without any regard to homogeneity in terms of the performance measures when applying a timing plan to that state.

Previous research also looked into using genetic algorithms for determining the TOD break points (Park et al. 2004). Such an approach looks at determining the time at which a different timing plan is activated. While the approach solves the problem of determining a threshold in volume to when the timing plan needs to be switched, it does not address higher states dimensionality nor coordination effects.

\section{Proposed Approach}

The proposed approach consists of the following steps:

1. Design timing plans for all significant levels of state conditions. In this context, significant level increase is an in

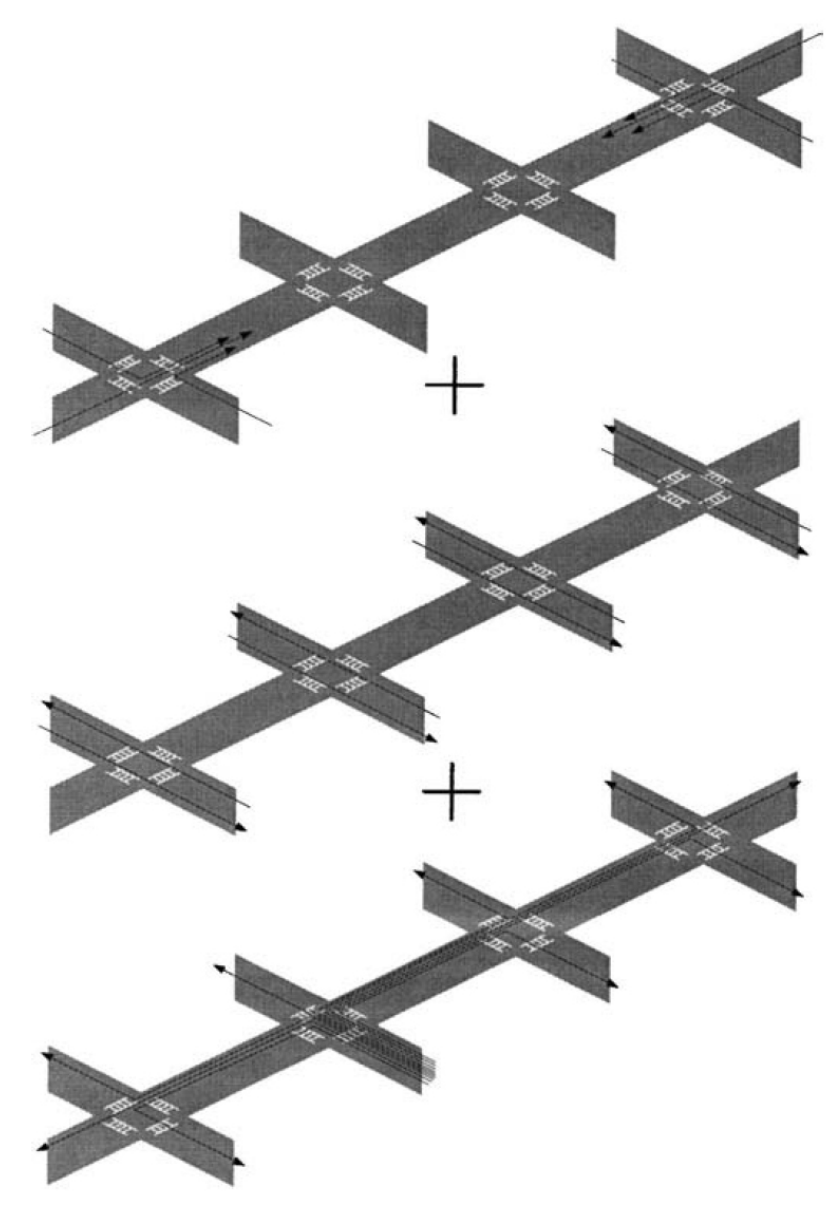

Figure 1. Generalized arterial volume distribution

crease in number of vehicles that could result in more than 2 seconds difference in phase duration as obtained from a signal optimization program (such as PASSER V). For example, one vehicle per cycle increase in a left turn phase could be translated as 60 vehicles/hour assuming a 60 second cycle length. Actual levels used in the analysis will be described later. 2. Run each timing plan with all traffic states in a batch mode to obtain delay and stop matrices for all plan-state combinations. This step was performed using the PASSER $\mathrm{V}$ optimization package.

3. In addition to the traditional performance measures (stops and delay), define a new performance measure known as the degree of detachment (DOD). The DOD measures the degree by which a traffic state is detached from adjacent states. In this context, detachment occurs when the adjacent state (the state that has one level below or one level above the current state's level) is associated with a different timing plan.

4. Conduct a multiobjective optimization for the delay, stops, and DOD using a nondominated sorting genetic algorithm. The algorithm produces $n$ number of timing plans and traffic state-timing plan association that results in the least delay and least number of stops, while maintaining that timing plans are assigned to mostly adjacent states. This makes it easier for the engineer to define thresholds based on volume to switch to another timing plan. 
Table 1. Volume Levels for Arterial External Movements

External movement

\begin{tabular}{lrrrrrr}
\hline Level & $\begin{array}{c}\text { East bound } \\
\text { (thru) }\end{array}$ & $\begin{array}{c}\text { South bound } \\
\text { (left) }\end{array}$ & $\begin{array}{c}\text { North bound } \\
\text { (right) }\end{array}$ & $\begin{array}{c}\text { West bound } \\
\text { (thru) }\end{array}$ & $\begin{array}{c}\text { North bound } \\
\text { (left) }\end{array}$ & $\begin{array}{c}\text { South bound } \\
\text { (right) }\end{array}$ \\
\hline 1 & 400 & 0 & 0 & 400 & 0 & 0 \\
2 & 800 & 200 & 200 & 800 & 200 & 200 \\
3 & 1,200 & 300 & 300 & 1,200 & 300 & 300 \\
4 & 1,600 & - & - & 1,600 & - & - \\
\hline
\end{tabular}

\section{Network Geometry and Timing Plan Generation}

In order to cover all reasonable traffic states in this analysis, a global perspective was used to look at all possible traffic states. The global perspective classifies arterial volume into three main movements as shown in Figure 1: (1) major external movement to the arterial; (2) additional cross-street movement; and (3) internal local movements. Preliminary PASSER runs were conducted to find the realistic limit of each movement in a four-intersection system so that the intersections are not oversaturated. The levels for each external movement are shown in Table 1. Equal cross-street demand was assumed at each level. The reader could look at the equal demand on the cross street as the maximum expected demand. This is only important at a strategic level to calculate the system timing plans, assuming that a fieldactuated controller handles the difference in demand at a tactical level. Levels and resulting interior turning volumes are shown in Table 2. These internal turning levels were obtained from a balanced origin-destination analysis, assuming equal attraction at each destination. The global perspective resulted in the formation of 3,888 traffic states $(4 \times 3 \times 3)$ east bound external movement $\times(4 \times 3 \times 3)$ west bound external movement $\times 3$ cross street levels. After eliminating all oversaturated conditions, 1,479 states remained. PASSER V was used to obtain the timing plans for each of these states for seven cycle lengths each $(60,75,90,100,120,150$, and 180 seconds). This resulted in 10,353 available timing plans. PASSER V was then run to evaluate the performance of each of these timing plans with each of the original states and two matrices of delay and number of stops were obtained for each of the state-plan combinations to be used as an input for the genetic algorithm (GA) optimization.

Table 2. Volume Levels for Internal Local Movements
Genetic Algorithms

GAs are optimization techniques based on the process of natural selection and genetics (Goldberg 1998). The GAs start by randomly selecting $n$ timing plans that constitutes the initial chromosomes. Through natural selection and the genetic operators, crossover, and mutation, chromosomes (sets of plans) with a better fitness (lower performance measures) are found. This natural selection process guarantees that chromosomes with the best fitness will propagate in future populations. The crossover operator mates genes (individual plans) from two parent chromosomes to form two new children chromosomes that have a high probability of having better finess than their parents. The crossover operator emphasizes the exploitation of the solution surface while the mutation operator allows new areas of the response surface to be explored, and prevents the solution from being trapped at local minima.

While GAs were widely used to solve traffic signal problems (Abu-Lebdah and Benekohal 2000), most of the previous research concentrated on either the optimization of one objective, or the optimization of several criteria by eventually integrating them into one objective.

\section{Nondominated Sorting Genetic Algorithm}

Nondominated sorting genetic algorithm (NSGA-II) belongs to a set of multiobjective algorithms that strive to find the Pareto front (the front of compromised solutions) of all objectives rather than integrating all objectives together (Dep et al. 2002). Solutions lying above the Pareto front are nonoptimal solutions, while those lying below the Pareto front are infeasible solutions. All solutions on the Pareto front are optimal with regard to a given objective weight preference

\begin{tabular}{|c|c|c|c|c|c|c|c|c|c|c|c|c|c|c|}
\hline \multirow{3}{*}{$\begin{array}{l}\text { Cross } \\
\text { street level }\end{array}$} & \multirow[b]{3}{*}{ Volume } & \multirow[b]{3}{*}{ Intersection } & \multicolumn{12}{|c|}{ Direction } \\
\hline & & & \multicolumn{3}{|c|}{ East bound } & \multicolumn{3}{|c|}{ West bound } & \multicolumn{3}{|c|}{ North bound } & \multicolumn{3}{|c|}{ South bound } \\
\hline & & & Left & Thru & Right & Left & Thru & Right & Left & Thru & Right & Left & Thru & Right \\
\hline \multirow[t]{4}{*}{1} & 150 & 1 & 21 & 127 & 21 & 134 & 113 & 134 & 131 & 19 & 19 & 19 & 19 & 131 \\
\hline & & 2 & 59 & 272 & 59 & 96 & 267 & 96 & 94 & 19 & 56 & 56 & 19 & 94 \\
\hline & & 3 & 96 & 267 & 96 & 59 & 272 & 59 & 56 & 19 & 94 & 94 & 19 & 56 \\
\hline & & 4 & 134 & 113 & 134 & 21 & 127 & 21 & 19 & 19 & 131 & 131 & 19 & 19 \\
\hline \multirow[t]{4}{*}{2} & 300 & 1 & 42 & 253 & 42 & 267 & 225 & 267 & 263 & 38 & 38 & 38 & 38 & 263 \\
\hline & & 2 & 117 & 544 & 117 & 192 & 534 & 192 & 188 & 38 & 113 & 113 & 38 & 188 \\
\hline & & 3 & 192 & 534 & 192 & 117 & 544 & 117 & 113 & 38 & 188 & 188 & 38 & 113 \\
\hline & & 4 & 267 & 225 & 267 & 42 & 253 & 42 & 38 & 38 & 263 & 263 & 38 & 38 \\
\hline \multirow[t]{4}{*}{3} & $300+100$ & 1 & 42 & 253 & 42 & 267 & 225 & 267 & 263 & 138 & 38 & 38 & 138 & 263 \\
\hline & & 2 & 117 & 544 & 117 & 192 & 534 & 192 & 188 & 138 & 113 & 113 & 138 & 188 \\
\hline & & 3 & 192 & 534 & 192 & 117 & 544 & 117 & 113 & 138 & 188 & 188 & 138 & 113 \\
\hline & & 4 & 267 & 225 & 267 & 42 & 253 & 42 & 38 & 138 & 263 & 263 & 138 & 38 \\
\hline
\end{tabular}




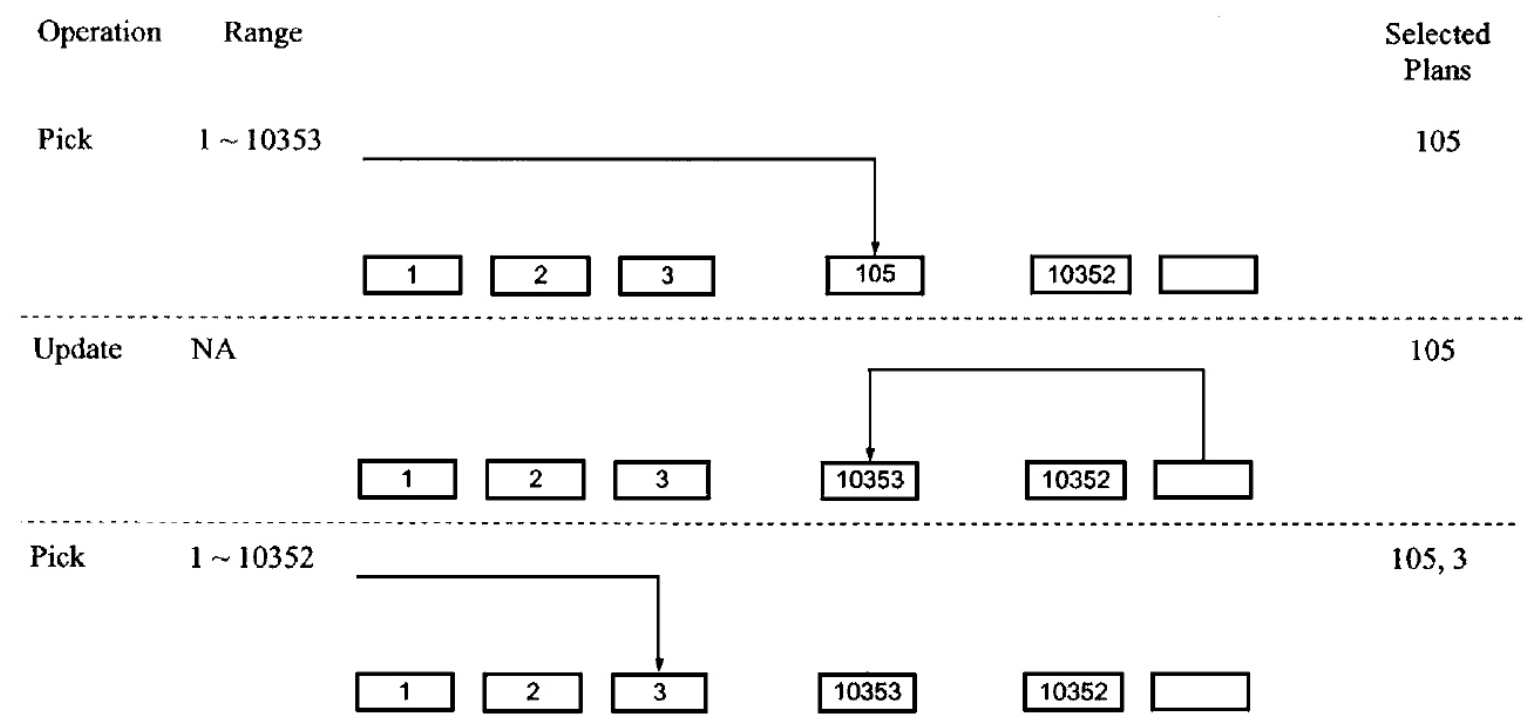

Fig. 2. Genetic algorithm selection of timing plans

set. The shape of the Pareto front itself provides very valuable information to theanalyst. Onewould know, looking at theParetofrontshape, how much other objectivefunctions would be compromised if a selected objective function is to be favored.

The NSGA-II is similar to simple GAs in the use of the selection, crossover, and mutation operators. However, prior to the selection step, the algorithm ranks the whole population based on all objectives. All individuals in the population that are nondominated (i.e., there does not exist an individual that is better than this individual in all objectives) are given a rank of one. These individuals with rank one are removed from consideration and all other individuals are ranked again and are assigned a rank of two. The process continues until all individuals are assigned a rank. After the process is completed, a crowding distance is calculated for all individuals. The crowding distance is used to diversify the population by assigning a higher value to individuals with larger cuboid formed by the individual and its neighboring individuals. The selection operator is then applied while assigning higher fitness to individuals with higher ranks and crowding distances. The algorithm ensures elitism by combining the parent population with the children population before the crossover and mutation operators are applied.

\section{Plan Selection Optimization}

The methods by which GA operators are applied would normally produce random individual values. However, in applying GAs to the selection of plans, it was necessary to satisfy two constraints: (1) each individual solution must contain only integer numbers within the range of the total number of plans and (2) the integer numbers must be unique within any individual. These two conditions had to be considered during the initialization of populations and the crossover and mutation operations.

In order to satisfy these requirements and uniquely select each chromosome gene (plan number), the selection routine in the GAs program used a consecutive integer array $r($ ) initially having values ranging between one and the total number of plans $(n)$. Restricting the plans to be coded as a set of consecutive integer numbers, the routine picks the first plan randomly by picking an integer number $i$ between 1 and $n$ and selecting the location that is in $r(i)$ position. The $r()$ array is then updated by setting the value of $r(i)$ to $r(n+$ $1-j)$, where $j$ is the number of plans selected up to the moment. The routine then chooses the second plan location by randomly picking an integer number within the range of 1 to $n-j$ as illustrated in Figure 2. Crossover and mutation were conducted in a similar fashion to ensure the production of valid chromosomes.

The same concept was applied to the optimization of the state-plan association. For each state, the GA assigned one of the plans included in the individual solution and assigned it to the particular state. Crossover and mutation were performed for the association as well. The fitness value of each individual solution was then calculated by looping through every state and accumulating the delay and stop from the state-plan look-up matrix. Although this approach could result in optimum system delay and stops, it does not address the clustering requirement that neighboring traffic states should preferably have similar timing plans. The clustering requirement had to be addressed with a new performance measure that is defined in the next section.

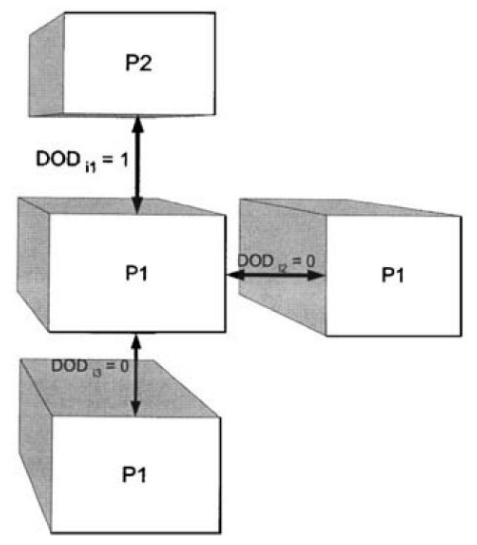

Fig. 3. DOD concept 

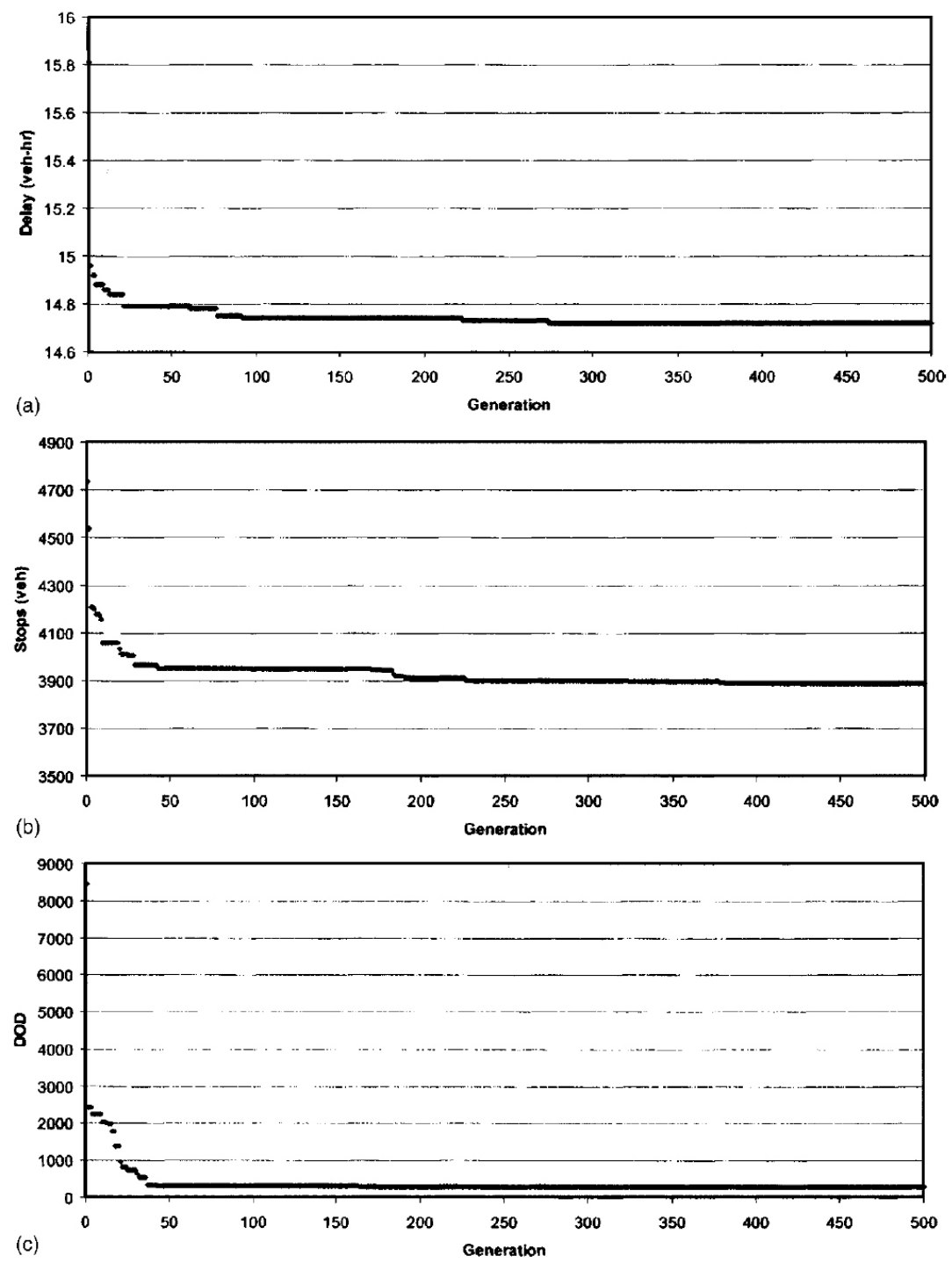

Fig. 4. GA evolution: (a) delay evolution; (b) stops evolution; and (c) DOD evolution

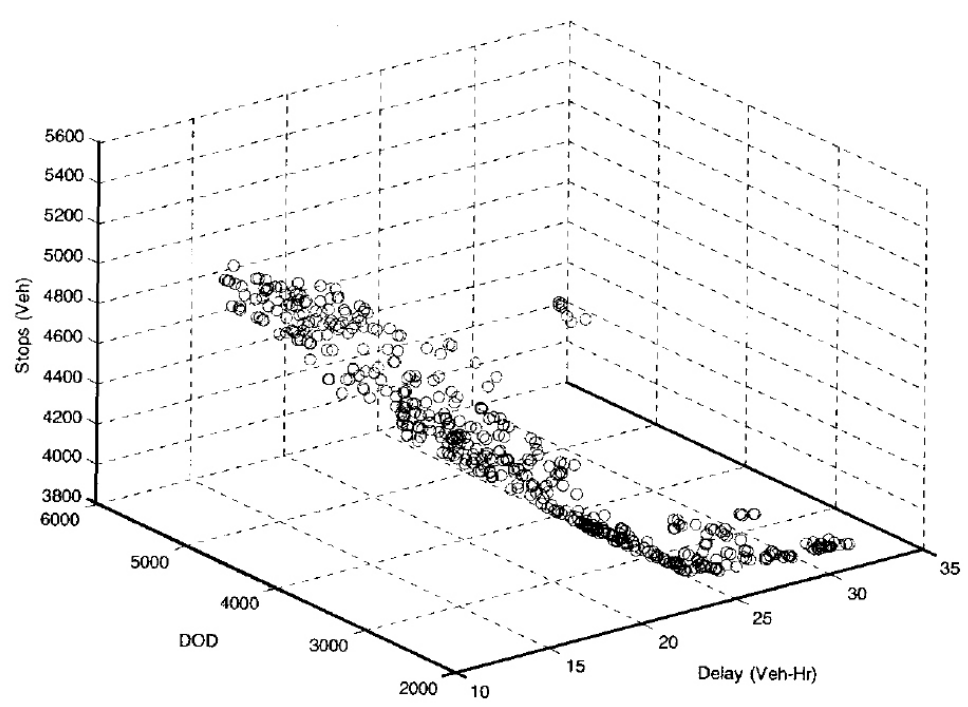

Fig. 5. Pareto front 

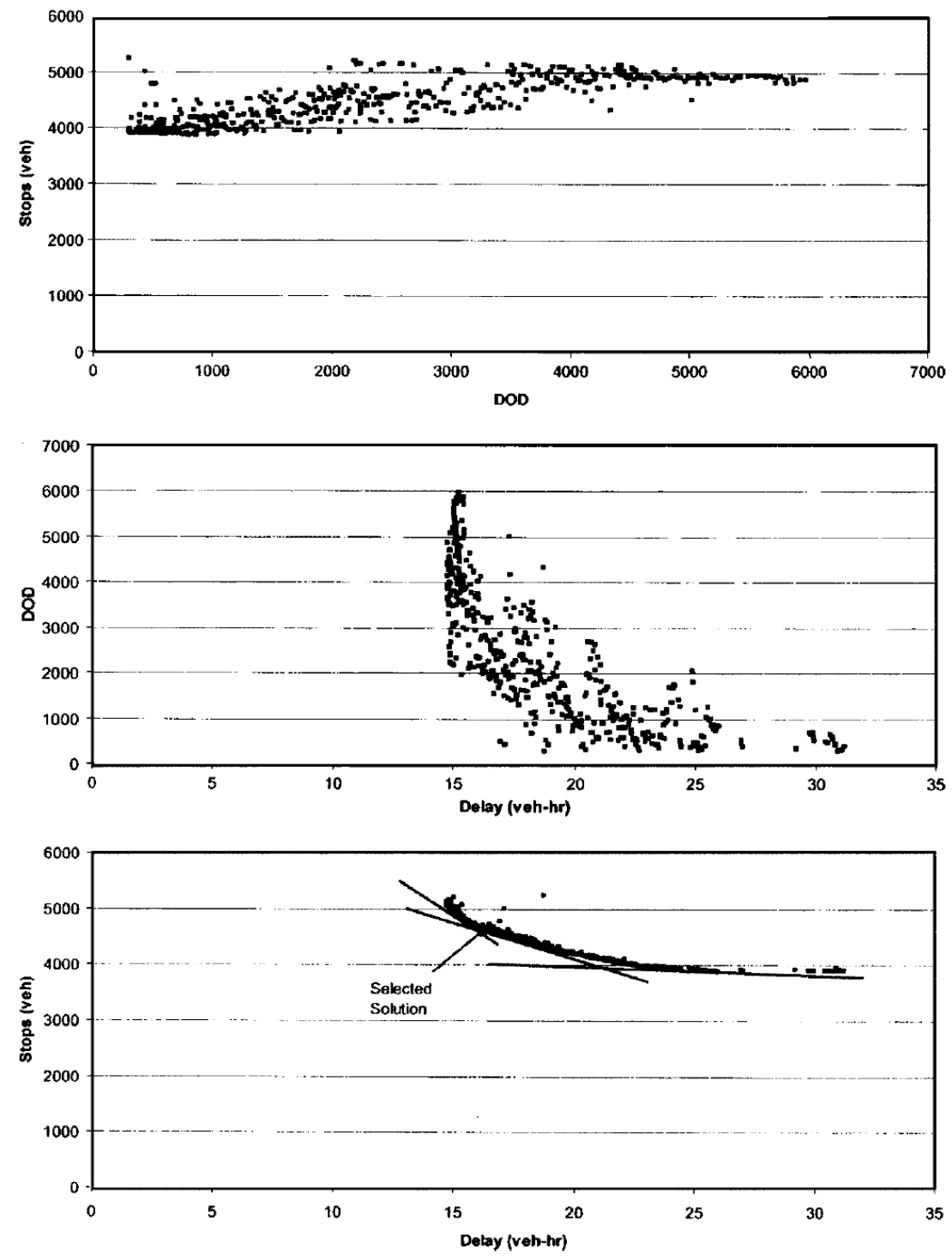

Fig. 6. Projections of Pareto front into two objective axis

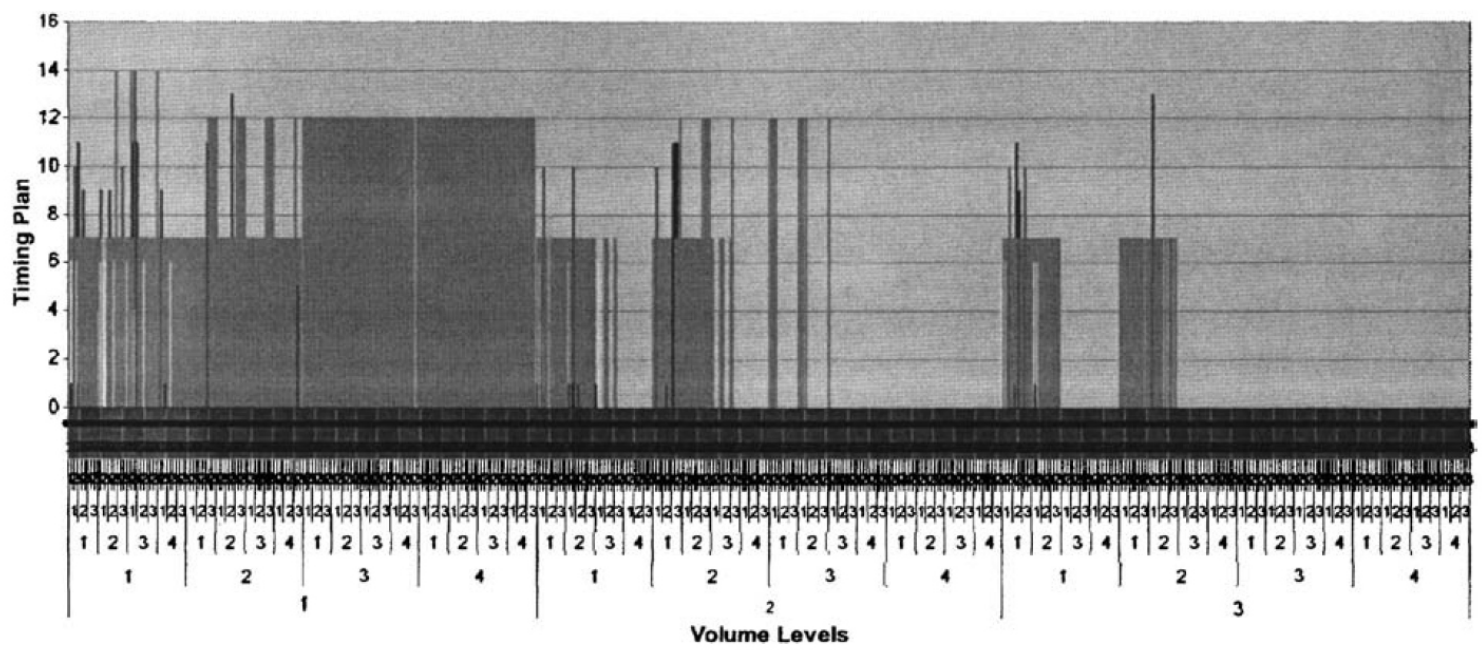

Fig. 7. Final clustering of traffic states 
Table 3. Final Selection of Timing Plans

\begin{tabular}{|c|c|c|c|c|c|c|c|c|c|c|c|c|}
\hline \multirow{2}{*}{$\begin{array}{l}\text { Timing } \\
\text { plan }\end{array}$} & \multirow[b]{2}{*}{ Intersection } & \multirow[b]{2}{*}{ Cycle } & \multicolumn{8}{|c|}{ Phase } & \multirow[b]{2}{*}{ Sequence } & \multirow[b]{2}{*}{ Offset } \\
\hline & & & 1 & 2 & 3 & 4 & 5 & 6 & 7 & 8 & & \\
\hline \multirow[t]{4}{*}{1} & 1 & 60 & 10 & 28 & 10 & 12 & 10 & 28 & 12 & 10 & Lead-Lead & 0 \\
\hline & 2 & 60 & 10 & 30 & 10 & 10 & 10 & 30 & 10 & 10 & Lag-Lead & 25 \\
\hline & 3 & 60 & 10 & 30 & 10 & 10 & 10 & 30 & 10 & 10 & Lead-Lag & 37 \\
\hline & 4 & 60 & 10 & 26 & 11 & 13 & 13 & 23 & 11 & 13 & Lag-Lead & 59 \\
\hline \multirow[t]{4}{*}{2} & 1 & 100 & 17 & 29 & 24 & 30 & 10 & 36 & 21 & 33 & Lead-Lead & 0 \\
\hline & 2 & 100 & 13 & 59 & 13 & 15 & 11 & 61 & 12 & 16 & Lag-Lead & 25 \\
\hline & 3 & 100 & 10 & 61 & 13 & 16 & 14 & 57 & 13 & 16 & Lag-Lead & 38 \\
\hline & 4 & 100 & 10 & 50 & 30 & 10 & 22 & 38 & 18 & 22 & Lead-Lag & 44 \\
\hline \multirow[t]{4}{*}{3} & 1 & 60 & 11 & 26 & 11 & 12 & 10 & 27 & 13 & 10 & Lead-Lead & 0 \\
\hline & 2 & 60 & 10 & 30 & 10 & 10 & 10 & 30 & 10 & 10 & Lag-Lead & 25 \\
\hline & 3 & 60 & 10 & 30 & 10 & 10 & 10 & 30 & 10 & 10 & Lead-Lag & 36 \\
\hline & 4 & 60 & 10 & 26 & 14 & 10 & 13 & 23 & 11 & 13 & Lead-Lead & 59 \\
\hline \multirow[t]{4}{*}{4} & 1 & 60 & 10 & 24 & 12 & 14 & 10 & 24 & 12 & 14 & Lead-Lead & 0 \\
\hline & 2 & 60 & 10 & 30 & 10 & 10 & 10 & 30 & 10 & 10 & Lag-Lead & 25 \\
\hline & 3 & 60 & 10 & 30 & 10 & 10 & 10 & 30 & 10 & 10 & Lead-Lag & 33 \\
\hline & 4 & 60 & 10 & 26 & 10 & 14 & 10 & 26 & 11 & 13 & Lead-Lead & 59 \\
\hline \multirow[t]{4}{*}{5} & 1 & 75 & 11 & 39 & 12 & 13 & 10 & 40 & 15 & 10 & Lead-Lead & 0 \\
\hline & 2 & 75 & 10 & 45 & 10 & 10 & 10 & 45 & 10 & 10 & Lead-Lead & 37 \\
\hline & 3 & 75 & 10 & 45 & 10 & 10 & 10 & 45 & 10 & 10 & Lead-Lag & 43 \\
\hline & 4 & 75 & 10 & 36 & 14 & 15 & 11 & 35 & 13 & 16 & Lag-Lead & 71 \\
\hline \multirow[t]{4}{*}{6} & 1 & 60 & 10 & 30 & 10 & 10 & 10 & 30 & 10 & 10 & Lead-Lead & 0 \\
\hline & 2 & 60 & 10 & 30 & 10 & 10 & 10 & 30 & 10 & 10 & Lag-Lead & 25 \\
\hline & 3 & 60 & 10 & 30 & 10 & 10 & 10 & 30 & 10 & 10 & Lead-Lag & 35 \\
\hline & 4 & 60 & 10 & 29 & 11 & 10 & 10 & 29 & 10 & 11 & Lag-Lead & 57 \\
\hline \multirow[t]{4}{*}{7} & 1 & 90 & 22 & 38 & 14 & 16 & 10 & 50 & 10 & 20 & Lead-Lead & 0 \\
\hline & 2 & 90 & 14 & 55 & 10 & 11 & 10 & 59 & 10 & 11 & Lag-Lead & 29 \\
\hline & 3 & 90 & 10 & 59 & 10 & 11 & 10 & 59 & 10 & 11 & Lead-Lag & 41 \\
\hline & 4 & 90 & 10 & 55 & 10 & 15 & 12 & 53 & 12 & 13 & Lead-Lag & 29 \\
\hline \multirow[t]{4}{*}{8} & 1 & 90 & 22 & 39 & 13 & 16 & 10 & 51 & 19 & 10 & Lead-Lead & 0 \\
\hline & 2 & 90 & 14 & 55 & 10 & 11 & 10 & 59 & 10 & 11 & Lag-Lead & 29 \\
\hline & 3 & 90 & 10 & 59 & 10 & 11 & 10 & 59 & 10 & 11 & Lead-Lag & 41 \\
\hline & 4 & 90 & 10 & 55 & 10 & 15 & 12 & 53 & 12 & 13 & Lead-Lag & 29 \\
\hline \multirow[t]{4}{*}{9} & 1 & 75 & 11 & 37 & 12 & 15 & 10 & 38 & 13 & 14 & Lead-Lead & 0 \\
\hline & 2 & 75 & 10 & 45 & 10 & 10 & 10 & 45 & 10 & 10 & Lag-Lead & 25 \\
\hline & 3 & 75 & 10 & 45 & 10 & 10 & 10 & 45 & 10 & 10 & Lead-Lag & 41 \\
\hline & 4 & 75 & 10 & 40 & 10 & 15 & 10 & 40 & 12 & 13 & Lag-Lead & 70 \\
\hline \multirow[t]{4}{*}{10} & 1 & 90 & 12 & 54 & 11 & 13 & 10 & 56 & 14 & 10 & Lead-Lead & 0 \\
\hline & 2 & 90 & 10 & 60 & 10 & 10 & 10 & 60 & 10 & 10 & Lead-Lag & 2 \\
\hline & 3 & 90 & 10 & 60 & 10 & 10 & 10 & 60 & 10 & 10 & Lag-Lead & 33 \\
\hline & 4 & 90 & 10 & 53 & 17 & 10 & 12 & 51 & 12 & 15 & Lead-Lead & 46 \\
\hline 11 & 1 & 90 & 18 & 32 & 18 & 22 & 10 & 40 & 10 & 30 & Lead-Lead & 0 \\
\hline & 2 & 90 & 14 & 46 & 14 & 16 & 12 & 48 & 13 & 17 & Lag-Lead & 25 \\
\hline & 3 & 90 & 10 & 50 & 13 & 17 & 16 & 44 & 14 & 16 & Lag-Lead & 25 \\
\hline & 4 & 90 & 10 & 41 & 10 & 29 & 23 & 28 & 18 & 21 & Lead-Lag & 43 \\
\hline 12 & 1 & 75 & 15 & 27 & 15 & 18 & 10 & 32 & 15 & 18 & Lead-Lead & 0 \\
\hline & 2 & 75 & 11 & 39 & 11 & 14 & 10 & 40 & 11 & 14 & Lead-Lead & 26 \\
\hline & 3 & 75 & 10 & 40 & 11 & 14 & 13 & 37 & 11 & 14 & Lead-Lag & 29 \\
\hline & 4 & 75 & 10 & 33 & 10 & 22 & 20 & 23 & 15 & 17 & Lag-Lead & 60 \\
\hline 13 & 1 & 75 & 15 & 26 & 16 & 18 & 10 & 31 & 18 & 16 & Lead-Lead & 0 \\
\hline & 2 & 75 & 11 & 40 & 11 & 13 & 10 & 41 & 11 & 13 & Lead-Lead & 26 \\
\hline & 3 & 75 & 10 & 40 & 11 & 14 & 13 & 37 & 11 & 14 & Lead-Lag & 28 \\
\hline & 4 & 75 & 10 & 33 & 17 & 15 & 20 & 23 & 15 & 17 & Lag-Lead & 60 \\
\hline
\end{tabular}


Table 3. (Continued.)

\begin{tabular}{|c|c|c|c|c|c|c|c|c|c|c|c|c|}
\hline \multirow{2}{*}{$\begin{array}{l}\text { Timing } \\
\text { plan }\end{array}$} & \multirow[b]{2}{*}{ Intersection } & \multirow[b]{2}{*}{ Cycle } & \multicolumn{8}{|c|}{ Phase } & \multirow[b]{2}{*}{ Sequence } & \multirow[b]{2}{*}{ Offset } \\
\hline & & & 1 & 2 & 3 & 4 & 5 & 6 & 7 & 8 & & \\
\hline \multirow[t]{4}{*}{14} & 1 & 90 & 19 & 23 & 19 & 29 & 10 & 32 & 19 & 29 & Lead-Lead & 0 \\
\hline & 2 & 90 & 14 & 41 & 14 & 21 & 12 & 43 & 12 & 23 & Lag-Lead & 25 \\
\hline & 3 & 90 & 10 & 44 & 13 & 23 & 17 & 37 & 14 & 22 & Lag-Lead & 23 \\
\hline & 4 & 90 & 10 & 35 & 11 & 34 & 20 & 25 & 18 & 27 & Lead-Lag & 42 \\
\hline
\end{tabular}

\section{Degree of Detachment}

The writers defined a new performance measure for the purpose of clustering traffic states. The DOD measures the degree by which a traffic state is detached from adjacent states. In this context, detachment occurs when the adjacent state (state that has a level one below or one above the current state's level) is associated with a different timing plan. Recall from Table 2 that any traffic state is basically represented by a seven element vector (three east bound external movement levels, three west bound external movement levels, and one cross street movement level). As such, there are 14 DOD degrees of freedom for any given state (seven upper levels and seven lower levels). Figure 3 shows an example of a DOD value for three levels in the state representation vector.

\section{Multiobjective Optimization Results NSGA-II Runs}

The NSGA-II was run to optimize the three objectives: delay, stops, and DOD. Different GA parameters were tried to investigate the shape of the Pareto front. Crossover probabilities tried were 0.8 and 0.9 . Population sizes tried were 80,200 , and 500. The mutation probabilities tried were 0.02 and 0.1. Most of the combinations produced similar Pareto fronts. Figure 4 shows the evolution of the solutions for delay, stops, and DOD, respectively. It can be deduced from Figure 4 that the solution converges around the 200th generation.

\section{Pareto Front}

The Pareto front obtained from the NSGA-II run is shown in Figure 5. The figure clearly shows the tradeoffs between different objectives. In order to simplify the selection of the final solution, the Pareto front was projected into three twoaxis figures as shown in Figure 6. It was clear from Figures $6(\mathrm{a}$ and $\mathrm{b})$ that solutions with low DOD values can be selected without significant adverse effects on the delay and stops. Figure 6(c) shows the effect of reducing the number of stopped vehicles on the overall delay on the system. The three lines show three different trends or "preferences." The most upper line would be the regions entertained by an analyst who is mainly pro delay reduction and does not put a significant weight on the objective of minimizing vehicular stops. The lower line would be the preferred region for an analyst interested mainly in minimizing vehicular stops without much regard to the huge delay incurred as a result of saving a small number of stops. The line in the middle is the region for the conservatives. In this paper, the selected solution shown in Figure 6(c) was chosen because it provided low DOD and the least delay within the second region.

\section{State-Plan Association}

Figure 7 shows the timing plan number (shown on the $y$ axis) that is associated with each state, as defined by the combination of volume levels in each dimension of the traffic state (shown on the $x$-axis). It can be seen in the figure that there is a clear clustering of traffic states. The breaking points of these clusters were chosen by the NSGA-II to produce minimum overall delay and number of vehicular stops.

Table 3 shows the final timing plans selected. Traffic states were associated with only 14 timing plans, with a resulting total average delay of 16.2 vehicles/hour and total average stops of 4,656 vehicles. Compared to the worst possible solutions encountered during the optimization of 34.22 vehicles/hour and 5,722 stops (and not the worst possible solutions obtained by reversing the objective function, which would be much worse), the final solution provides a concurrent saving of $52.7 \%$ in delay and $18.6 \%$ in stops.

\section{Conclusion}

This paper introduced a new methodology for selection of optimal timing plans to be used with TRPS control. The paper addressed two of the most important challenges in setting up a TRPS system: (1) selection of optimal timing plans that are suitable for a wide range of traffic conditions and (2) association of each one of these traffic conditions to one of the few available timing plans while maintaining the clustering of traffic states together. The paper used a global perspective of looking at traffic states and used the NSGAII algorithm, with a newly defined performance measure, to achieve its objectives. Fourteen timing plans were identi.ed to provide optimal control of the traffic system with an average saving of at least $53 \%$ in delay and 19\% in number of vehicle stops.

\section{Acknowledgments}

This work was sponsored by the Texas Department of Transportation (TxDOT) and was conducted for TxDOT's Research and Technology Implementation Office. The materials and methods presented were developed as part of TxDOT Project No. 0-4421, "A Simplified Approach for Selecting Optimal Traffic Responsive Control Parameters." The writers would like to recognize the project director, Mr. Henry Wickes, for his support and guidance throughout the duration of this project. 


\section{References}

Abbas, M. M., Chaudhary, N. A., Pesti, G., and Sharma, A. (2004). "Guidelines for determination of optimal traf.c responsive plan selection control parameters." Research Rep. No. 4421-2. Texas Transportation Institute, College Station, Tex.

Abu-Lebdah, G., and Benekohal, R. (2000). “Genetic algorithms for traffic signal control and queue management of oversaturated two-way arterials." Transportation Research Record 1727, Transportation Research Board, Washington, D.C., 61-67.

Chaudhary, N., Kovvali, V., Chu, C., and Alam, S. (2002). "Software for timing signalized arterials." Research Rep. No. 40201, Texas Transportation Institute, College Station, Tex.

Dep, K., Pratap, A., Agarwal, S., and Meyarivan, T. (2002). "A fast and elitist multiobjective genetic algorithm NSGA-II." IEEE Trans. Evol. Comput., 6.2., 181-197.

Fambro, D., Sangineni, S., Lopez, C., Sunkari, S., and Barnes, R. (1995). "Benefits of the Texas Traffic Light Synchronization (TLS) Grant Program II: Volume I. Executive summary and appendices A-C." Research Rep. No. 3010-1F, Vol. 1, Texas Transportation Institute, College Station, Tex.
Goldberg, D. (1989). Genetic Algorithms in Search, Optimization, and Machine Learning, Addison-Wesley, Reading, Mass.

Hanbali, R., and Fornal, C. (1998). "Methodology of evaluating the effectiveness of traffic-responsive system on intersection congestion and traffic safety." Transportation Research Record 1603, Transportation Research Board, Washington, D.C., 137-149.

Meyer, M. (1997). A Toolbox for Alleviating Traffic Congestion and Enhancing Mobility, Institute of Transportation Engineers, Washington, D.C.

Nelson, E., Abbas, M., Shoup, G., and Bullock, D. (2000). Development of Closed-Loop System Evaluation Procedures. FHWA/ IN/JTRP, Federal Highway Administration, McLean, Va.

Park, B., Santra, P., Yun, I., and Lee, D. (2004). "Optimization of time-of-day breakpoints for better traffic signal control." Proc., 83rd Transportation Research Board Annual Meeting, Transportation Research Board, Washington, D.C.

Sharma, A. (2004). “Determination of Traffic Responsive Plan Selection Factors and Thresholds Using Artificial Neural Network." Master's thesis, Texas A\&M Univ., College Station, Tex. 